\title{
Background, foreground and nearby matter influence on strong gravitational lenses
}

\author{
M. Jaroszynski ${ }^{1 \star}$ and Z. Kostrzewa-Rutkowska ${ }^{1}$ \\ ${ }^{1}$ University of Warsaw Observatory, Al. Ujazdowskie 4, 00-478 Warsaw, Poland
}

Accepted 2012 April 26; Received 2012 April 25; in original form 2011 November 10

\begin{abstract}
We investigate strong lensing by non-singular finite isothermal ellipsoids taking into account the influence of the matter along the line of sight and in the close lens vicinity. We compare three descriptions of light propagation: the full approach taking into account all matter inhomogeneities along the rays, the single plane approach, where we take into account the influence of the strong lens neighbours but neglect the foreground and background objects, and the single lens approach. In each case we simulate many strong lensing configurations placing a point source at the same redshift but in different locations inside the region surrounded by caustics. We further analyze configurations of four or five images. For every simulated strong lensing configuration we attempt to fit a simplified lens model using a single isothermal ellipsoid or a single isothermal ellipsoid with external shear. The single lens fits to configurations obtained in the full approach are rejected in majority of cases with $95 \%$ significance. For configurations obtained in the single plane approach the rejection rate is substantially lower. Also the inclusion of external shear in simplified modeling improves the chances of obtaining acceptable fits, but the problem is not solved completely. The quantitative estimates of the rates of rejection of simplified models depend on the required accuracy of the models, and we present few illustrative examples, which show that both matter close to the lens and matter along the rays do have important influence on lens modeling. We also estimate the typical value of the external shear and compare the fitted parameters of the simplified models with the parameters of the lenses used in the simulations.
\end{abstract}

Key words: gravitational lensing: strong and weak - large-scale structure of the Universe

\section{INTRODUCTION}

Strong gravitational lensing of distant sources has low probability and is usually attributed to a single concentration of matter close to the line of sight, while the influence of anything else is neglected (see e.g. Kochanek 2006 for a review of strong lensing). In some cases simple, single component models of lenses are not satisfactory. Even the first lensed quasar observed, QSO 0957+561 A,B (Walsh et al. 1979; Gunn et al. 1979) requires a combination of a galaxy and a galaxy cluster to model its basic properties Young et al. 1981).

The influence of another object along the line of sight on the main lens properties has been investigated by Kochanek \& Apostolakis (1988). Keeton et al. (1997) argue that adding an external shear to an elliptic lens model greatly improves the fits of four image configurations, but they conclude that the shear is probably related to the main lens. Bar-Kana (1996) investigates the influence of the density fluctuations along the line of sight on the accuracy of Hubble constant determination based on time delays measured in some of the multi-image QSO

^ E-mail: mj@astrouw.edu.pl (MJ); zkostrze@ astrouw.edu.pl (ZKR) systems. Chen et al. (2003) check the influence of the substructure not related to the main lens on the observed flux ratios in lensed images. Wambsganss et al. (2004) study the probability of giant arcs using the results of a large cosmological simulation and multi-layer approach to backward ray tracing. They find that the matter on the way slightly increases the probability of arcs. Wambsganss et al. (2005) address specifically the question of secondary lenses on the line of sight. According to this study the role of secondary lenses is a strong function of source redshift: it can be neglected in $95 \%$ of cases for a source at $z_{\mathrm{S}}=1$, but is important in $38 \%$ of cases at $z_{\mathrm{S}}=3.8$. Using single plane approach introduces systematic errors to the estimated lens parameters. In a recent paper D'Aloisio \& Natarajan (2011) investigate the influence of the secondary lenses in the line of sight on the accuracy of estimating the dark energy density.

It is probably impossible to quantify the influence of the matter along the line of sight and of the strong lens close (but possibly unobservable) neighbours in a single case of real observed multiple image configuration. In this paper we are going to obtain a statistical measure of such an influence performing simulations of many multiple image configurations using a realistic model of light propagation in an inhomogeneous Universe model. To find the role of 
matter not belonging to the strong lens, we follow the rays including or not the lens neighbours and inhomogeneities along the rays. Again, the direct comparison between individual image configurations obtained as a result of different descriptions of light propagation seems useless, since one should compare positions and flux ratios of images of a point source at the same position and it is not clear how to compare source positions between the different models of light propagation. If image configuration is considered, it is the source position relative to the caustic structure which counts and since the caustic structures are different the problem persists.

Instead of making comparisons on a one to one basis, we compare the properties of all four or five image configurations between the models of light propagation. If the influence of matter outside the strong lens were unimportant, each lens - image configuration would be similar to some configuration obtained with a single lens model. To check whether this is the case we attempt to fit such simplified model to all image configurations considered. The success rate of the fitting procedure applied to configurations obtained with a different model of light propagation statistically measures the similarity of this model to the single lens model.

In Sec. 2 we describe our models of light propagation. Sec. 3 presents tools used to compare different models and the results of such comparison. Discussion and conclusions follow in Sec. 4.

\section{MODEL OF THE LIGHT PROPAGATION}

\subsection{Deflection angles}

The results of the Millennium Simulation Springel et al. 2005) give the matter distribution (the positions and velocities of $\sim 10^{10}$ simulation particles) in the 64 epochs corresponding to the redshifts $0 \leqslant z_{i} \leqslant 127$. In our calculations we assume, that the propagation of a ray between redshifts $z_{i_{1}} \equiv\left(z_{i-1}+z_{i}\right) / 2$ and $z_{i_{2}} \equiv\left(z_{i+1}+z_{i}\right) / 2$ is affected by matter distribution given for the epoch $z_{i}$. We are interested in sources at high redshifts $(z \geqslant 1)$, so there are always many such matter layers on the ray path, and each of them can be considered thin. Thus the multilayer approach (Schneider \& Weiss 1988) with layers corresponding to the Millennium epochs is natural. The position of the ray in the $N$-th layer in the angular coordinates is given as

$\boldsymbol{\beta}_{N}=\boldsymbol{\beta}_{1}-\sum_{i=1}^{N-1} \frac{d_{i N}}{d_{N}} \boldsymbol{\alpha}_{i}\left(\boldsymbol{\beta}_{i}\right)$

where $d_{i N}$ is the angular diameter distance as measured by an observer at epoch $i$ to the source at epoch $N, d_{N}$ - the angular diameter distance to the same source measured by a present $(z=0)$ observer, and $\boldsymbol{\alpha}_{i}\left(\boldsymbol{\beta}_{i}\right)$ is the deflection angle in the $i$-th layer at the position $\boldsymbol{\beta}_{i}$. In a flat cosmological model the angular diameter distances in the lens equation can be replaced by comoving distances. Below we use comoving distances $D(z)$, denoting $D_{i} \equiv D\left(z_{i}\right)$ for short. Since the comoving distances are additive in a flat model, one has $d_{i N} / d_{N}=\left(D_{N}-D_{i}\right) / D_{N}$. In the calculations we apply more efficient recurrent formula of Seitz \& Schneider (1992), equivalent to the above equation.

To calculate the deflection angle in a given layer we need a description of its matter distribution. This is done in two steps. The averaged matter density is defined on a coarse grid of $256^{3}$ cells. The gravitationally bound haloes have been described by De Lucia \& Blaizot (2007) and Bertone et al. (2007). Both kinds of data are accessible from the Virgo - Millennium Database (Lemson \& Springel 2006). Jaroszynski \& Kostrzewa (2010) use the same information on matter distribution, but their calculation of lensing effect is based on different approach.

Since only the positions, virial masses, virial radii, and virial velocities of the haloes can be obtained from the Database, the detailed matter distributions of each halo must be supplemented. We use non singular isothermal ellipsoids (NIE) as models of individual lenses. The averaged matter density includes also the matter belonging to haloes, so the halo mass models must be compensated by a shallow negative density distribution (see below).

In constructing the matter layers we follow Carbone et al. (2008), randomly rotating and shifting simulation cubes corresponding to different epochs, which eliminates the consequences of periodic boundary conditions used in their calculations. The comoving thickness of the $i$-th layer $D_{i_{2}}-D_{i_{1}}$ is of the order of $10^{2} \mathrm{Mpc}$, always smaller than the simulation cube size $500 / \mathrm{h} \mathrm{Mpc}$. The 2D layers are not periodic in general. Hilbert et al. (2009) define specific projection directions to obtain periodic $2 \mathrm{D}$ density distributions and use 2D Fourier transforms in calculations of the deflection angles. Our 3D grid $\left(256^{3}\right)$ is small enough to employ spectral methods. Using Poisson equation we calculate 3D gravitational acceleration $\boldsymbol{g}$ on the grid. The deflection of a ray passing the $i$ th layer at position $\boldsymbol{\beta}_{i}$ due to the averaged (or background) matter distribution is given as:

$\boldsymbol{\alpha}_{i}^{\mathrm{bcg}}\left(\boldsymbol{\beta}_{i}\right)=\frac{2}{\left(1+z_{i}\right) c^{2}} \int_{D_{i_{1}}}^{D_{i_{2}}} \boldsymbol{g}_{\perp}\left(D, \boldsymbol{\beta}_{i}\right) d D$

where $\boldsymbol{g}_{\perp}$ is the component of gravitational acceleration perpendicular to the ray, and its value at any location is obtained by the interpolation on the $3 \mathrm{D}$ grid. $(D, \boldsymbol{\beta})$ are used as coordinates. Since our simulated maps of the sky cover typically regions of a few minutes of arc in size, the position components $\left(D \beta_{x}, D \beta_{y}\right)$ may be treated as Cartesian coordinates in the plane perpendicular to the propagation direction, and the comoving distance $D$ serves as radial coordinate. Because the integration should be performed over the proper distance, instead of comoving one, the result is divided by the factor $1+z_{i}$.

For each individual halo we apply the model of non-singular isothermal ellipsoid as described by Kormann et al. (1994). The deflections in 2D real notation are given by Kochanek (2006):

$\alpha_{x}=\frac{\alpha_{0}}{q^{\prime}} \arctan \left(\frac{q^{\prime} x}{\omega+r_{0}}\right) \quad \alpha_{y}=\frac{\alpha_{0}}{q^{\prime}} \operatorname{artanh}\left(\frac{q^{\prime} y}{\omega+q^{2} r_{0}}\right)$

where:

$$
\omega\left(x, y, q, r_{0}\right)=\sqrt{q^{2}\left(x^{2}+r_{0}^{2}\right)+y^{2}} \quad q^{\prime}=\sqrt{1-q^{2}}
$$

The ray crosses the lens plane at $(x, y)$, the lens centre is placed at the origin of the coordinate system, the major axis along $x$. The axis ratio is given by $q, r_{0}$ is the core radius, and $\alpha_{0}$ is the deflection angle parameter (see below).

The convergence $\kappa$ (which is proportional to the surface mass density) has the required (NIE) form:

$\kappa\left(x, y, q, r_{0}\right)=\frac{1}{2}\left(\alpha_{x, x}+\alpha_{y, y}\right)=\frac{1}{2} \frac{\alpha_{0}}{\omega\left(x, y, q, r_{0}\right)}$

Far from the centre the 2D matter distribution becomes elliptical, as required.

The NIE matter distribution is infinite with divergent total mass. To obtain a finite mass lens we follow Kochanek (2006) combining two NIE distributions with different core radii $r_{1} \ll r_{2}$. Inside $r_{1}$ the surface density is approximately constant, isothermal in between, and sharply falling outside $r_{2}$ : 


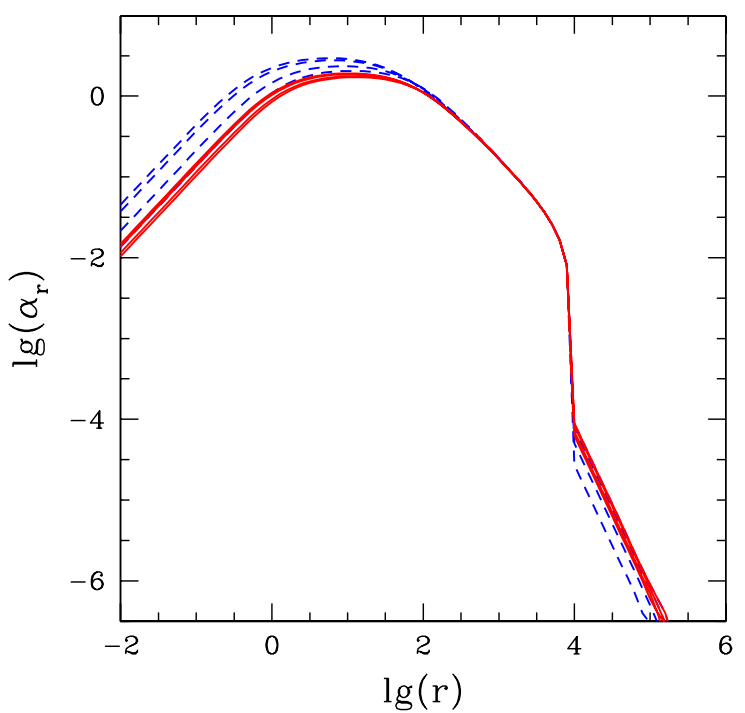

Figure 1. The deflection angle of the compensated lens. For illustration we use: $r_{1}=1, r_{2}=10^{2} r_{1}$, and $r_{\text {lim }}=10^{2} r_{2}$. The plots show the radial deflection component $\alpha_{r}$ as function of radius for fixed polar angle $\phi=90^{\circ}$, $60^{\circ}, 30^{\circ}$, and $0^{\circ}$ (top - down). Dashed lines are used for $q=0.3$, and solid lines for $q=0.7$.

$\kappa(x, y, q)=\kappa\left(x, y, q, r_{1}\right)-\kappa\left(x, y, q, r_{2}\right) \rightarrow \frac{1}{4} \frac{\alpha_{0}\left(r_{2}^{2}-r_{1}^{2}\right)}{\omega^{3}(x, y, q, 0)}$

which gives a non-singular, finite mass lens with the deflection angle:

$\boldsymbol{\alpha}(x, y, q)=\boldsymbol{\alpha}\left(x, y, q, r_{1}\right)-\boldsymbol{\alpha}\left(x, y, q, r_{2}\right) \rightarrow \alpha_{0}\left(r_{2}-r_{1}\right) \frac{\boldsymbol{r}}{r^{2}}$

For large radii $r \gg r_{2}$ the lens acts as a spherical finite mass contained within $r_{2}$. We identify the mass and radius of the lens with the virial mass and radius of the halo, so the characteristic deflection angle is given as:

$\alpha_{0}=\frac{4 G M_{\mathrm{vir}}}{c^{2}\left(r_{2}-r_{1}\right)} \approx \frac{4 G M_{\mathrm{vir}}}{c^{2} r_{\mathrm{vir}}} \quad r_{2}=r_{\mathrm{vir}}$

To compensate the lens we use a circular disk of radius $r_{\text {lim }} \gg$ $r_{\text {vir }}$ with constant surface density and with the deflection angle:

$\boldsymbol{\alpha}_{\mathrm{cmp}}(\boldsymbol{r})=\frac{4 G M_{\mathrm{vir}}}{c^{2}} \times \begin{cases}\boldsymbol{r} / r_{\lim }^{2} & \text { if } r \leqslant r_{\text {lim }} \\ \boldsymbol{r} / r^{2} & \text { if } r>r_{\text {lim }}\end{cases}$

where

$M_{\mathrm{vir}} \equiv \frac{4}{3} \pi \rho_{0} r_{\mathrm{lim}}^{3}$

defines the size of the disk. $\rho_{0}$ is the average matter density in the Universe at the lens redshift. In our approach we always use $r_{1}=$ $0.01 r_{2}$, so we avoid singularity at the lens centre, but we do not assign any physical meaning to this parameter.

Finally we check numerically the dependence of the deflection angle on various parameters. We use the formula for a compensated lens:

$\boldsymbol{\alpha}(\boldsymbol{r})=\boldsymbol{\alpha}\left(x, y, q, r_{1}\right)-\boldsymbol{\alpha}\left(x, y, q, r_{2}\right)-\boldsymbol{\alpha}_{\mathrm{cmp}}(\boldsymbol{r})$

We illustrate the dependence of deflection angle on position in Fig. 1. using arbitrary units and a case with $r_{1} \ll r_{2} \ll r_{\text {lim. }}$. The compensation is not exact, but at $r \approx r_{\text {lim }}$ the deflection angle drops by two orders of magnitude.

A compensated lens has only a finite range. Its radius depends on the properties of each individual halo, but investigating the deflection by haloes at any position in a given layer we take into account only the finite number of lenses:

$\boldsymbol{\alpha}_{i}^{\text {haloes }}(\boldsymbol{\beta})=\sum_{j} \boldsymbol{\alpha}\left(\boldsymbol{\beta}-\boldsymbol{\beta}_{j}\right)$ where : $D_{i}\left|\boldsymbol{\beta}-\boldsymbol{\beta}_{j}\right| \leqslant r_{j}^{\lim }$

The expression $\boldsymbol{\alpha}\left(\boldsymbol{\beta}-\boldsymbol{\beta}_{j}\right)$ stands for the deflection at the position $\boldsymbol{\beta}$ caused by the lens at position $\boldsymbol{\beta}_{j}$ within its limiting range.

The total deflection in the $i$-th layer

$\boldsymbol{\alpha}_{i}\left(\boldsymbol{\beta}_{i}\right)=\boldsymbol{\alpha}_{i}^{\text {bcg }}\left(\boldsymbol{\beta}_{i}\right)+\boldsymbol{\alpha}_{i}^{\text {haloes }}\left(\boldsymbol{\beta}_{i}\right)$

can be calculated for a ray passing at any location $\boldsymbol{\beta}_{i}$.

\subsection{Maps of the sky}

We calculate deflection angles for all layers of interest storing them on the $2048^{2}$ grids with 0.1 arcsec resolution. Using a set of stored deflection angles for all layers and interpolating on the grids one can apply Eq.(1), mapping a region of $\approx 3^{\prime} \times 3^{\prime}$ in the sky. We investigate light propagation from sources at $z_{S} \approx 2$ taking into account the influence of several thousands haloes in the field of view and some in adjacent regions, if they are within their $r_{\text {lim }}$ ranges. We construct eight separate maps pointing in random directions.

When investigating multiple image properties we need zoomed maps of smaller parts of the sky. For better resolution we use finer grids with deflections interpolated from previous calculations with the help of bi-cubic spline, so the interpolated deflection derivatives are continuous. The backward ray shooting on a finer grid is repeated.

Treating each of the individual haloes in the field of view as an isolated lens of known velocity dispersion and using SIS model we calculate its Einstein radius $r_{E}$ for a source at given redshift $z_{S}$ in a homogeneous Universe model. In each simulated map of the sky we find 10 lenses with the largest Einstein radii. We expect that these dominating lenses have the highest probability of producing multiple images.

In the single SIS lens case the second image is possible only for a source within the Einstein ring. The brighter of the two images lies within $2 r_{E}$ from the lens centre and the dimmer within $1 r_{E}$, so we expect that in more complicated problem, including lens ellipticity and the influence of other lenses the multiple images lie within few Einstein radii from each other.

We check for the presence of other haloes inside the circle of the radius $3 r_{E}$ surrounding a dominating lens. If they are present we enlarge the region of interest including $3 r_{E}$ zones around all companions. Finally we repeat backward ray shooting inside a square on the sky including the region of interest. The fine grids giving the deflection angles in consecutive layers encompass still larger areas, so one can follow rays deflected off the main region. The result of the ray shooting is a vector array:

$\boldsymbol{\beta}_{N}^{k l}=\boldsymbol{\beta}_{N}\left(\boldsymbol{\beta}_{1}^{k l}\right)$

where $\boldsymbol{\beta}_{N}^{k l}$ gives the positions in the source plane of rays apparently coming from the directions $\boldsymbol{\beta}_{1}^{k l}$ on the observer's sky. Superscripts $k, l$ enumerate the rays. The $\boldsymbol{\beta}_{N}\left(\boldsymbol{\beta}_{1}\right)$ relation is given in Eq.(1). 

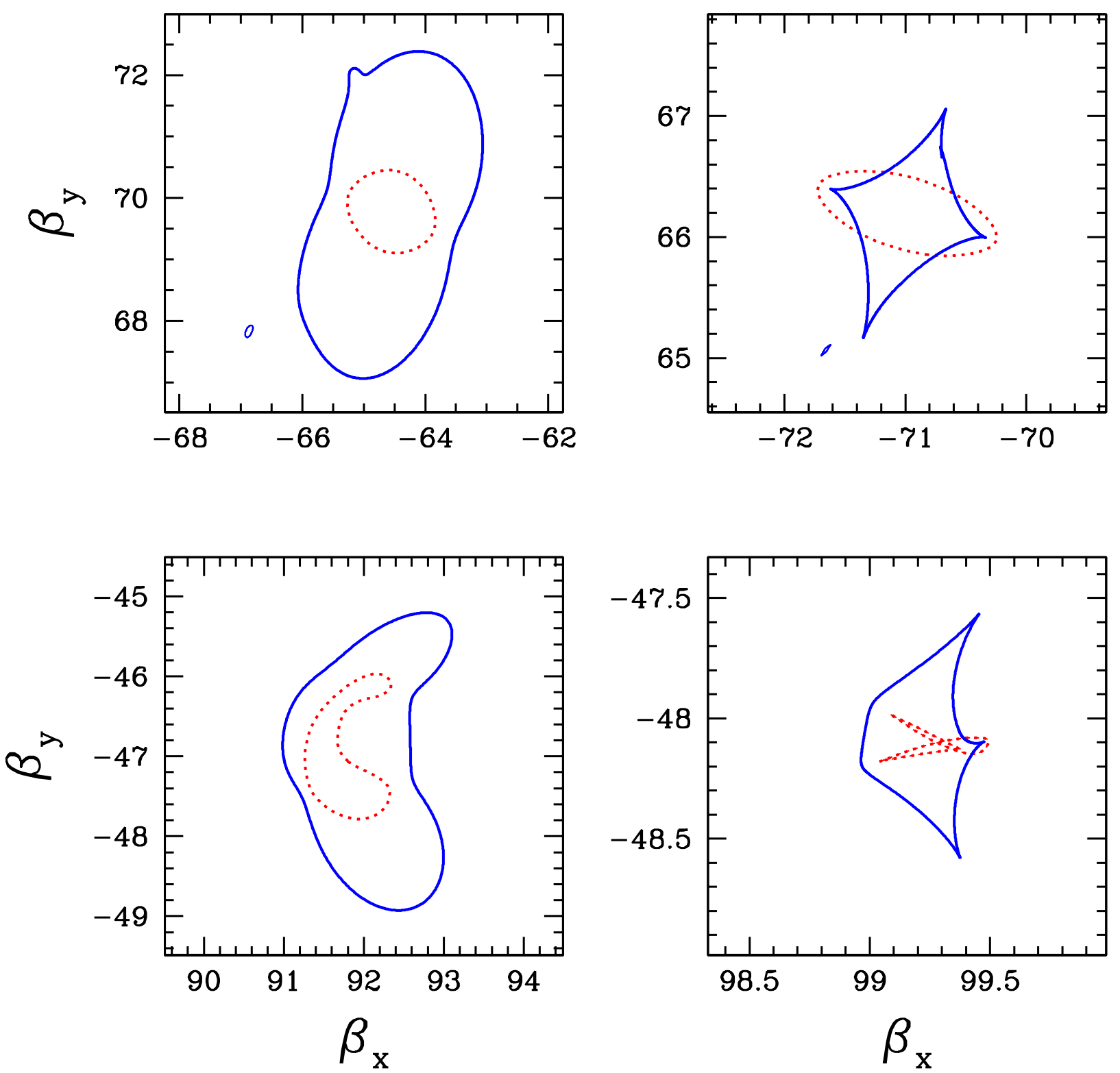

Figure 2. Two examples of critical lines and caustic structures obtained using Case 1 light propagation model. Critical lines are shown in the left column. The coordinates are relative to the centre of $3^{\prime} \times 3^{\prime}$ maps and are expressed in arcseconds. The corresponding caustic structures are shown on the right. The perturbations of the caustic structure by the surrounding haloes are apparent in the example shown in the upper row. In the lower row the action of two close elliptical lenses of similar masses, having different position angles completely changes the shapes of critical lines and caustics.

\subsection{Three propagation models}

To estimate the influence of the foreground and background matter in the beam and also of the lens neighbours belonging to the same redshift layer we consider three scenarios for the backward ray shooting. Very similar method is employed by Puchwein \& Hilbert (2009). In the full approach (Case 1) we include deflection of rays by matter in all layers between the source and observer, as described above. In the one layer approach (Case 2) we include deflection by all matter in the layer of dominating lens only, neglect- ing the influence of other layers. In the single lens approach (Case 3 ) only the deflection by the dominant lens is taken into account.

\subsection{Image finding}

To find critical lines in the sky we solve the standard equation (Schneider et al. 1992):

$$
\operatorname{det} \mathrm{A}=0 \quad \mathrm{~A} \equiv\left\|\frac{\partial \boldsymbol{\beta}_{N}}{\partial \boldsymbol{\beta}_{1}}\right\|
$$


where $A$ is the deformation matrix. The equation is solved approximately on the grid and then refined by iterations. Finding of caustic lines in the source plane is then straightforward.

Usually the critical lines and caustic structures obtained in three Cases of backward ray shooting are similar and the influence of matter outside the dominating lens is not apparent. There are however several cases showing the influence of smaller mass haloes on the critical lines and (to a lesser extent) caustics as shown in the upper row of Fig. 2 An example of a strong caustic perturbation is shown in the lower row of Fig. 2 .

We start image finding using approximate methods on the grid. We replace the point source by an extended surface brightness profile with the Gaussian shape and the characteristic radius of few pixels. The related surface luminosity in the sky is given by:

$I^{\mathrm{obs}}\left(\boldsymbol{\beta}_{1}\right)=I^{\mathrm{src}}\left(\boldsymbol{\beta}_{N}\left(\boldsymbol{\beta}_{1}\right)\right)$

The local maxima of the observed surface luminosity are the positions of the images of the source centre. We find brightness maxima on the grid and use them as approximate solutions to the lens equation. The improved positions are obtained by iterations.

Using the above method we find multiple images of point sources placed behind lenses. For each lens we try few hundreds to several thousands source positions (depending on the size of the caustics region) distributing them evenly. Each position considered represents the same surface area in the source plane. Finding a source at any location has the same probability if they are distributed randomly. (We neglect here the amplification bias caused by the strong lensing.) The procedure of image finding for many source positions is repeated for each of the ten strongest lenses belonging to each of the eight simulated maps of the small fragments of the sky obtained with the three different models of light propagation.

\section{FITTING THE SIMULATED STRONG LENSES WITH SIMPLIFIED MODELS}

In the case of pure elliptical, non-singular lens one would expect (in general) 1, 3, or 5 images. Our numerical method using at the beginning a small extended source and having finite resolution may miss some of the images. (See the Discussion). In Cases 1,2 the external influence may also change the number of images. Since configurations with more images probably better constrain the lens, we concentrate on cases with five or four images.

To estimate the influence of the galaxies in the lens vicinity and the matter along the line of sight on the properties of the strong lensing, we attempt to model all our simulated cases of multiple imaging using a single lens model in a uniform Universe. The single lens we are using in modeling is a non-singular finite isothermal ellipsoid used also in the simulations for each of the haloes. When tracing the rays in simulations we interpolate all deflection angles from earlier computed arrays. Single lens modeling uses analytically calculated deflection angles and their derivatives, so it serves also as a test of ray tracing simulations in Case 3.

We also try a more sophisticated lens model using the same non-singular finite isothermal ellipsoids with external shear. In this way the tidal influence of masses close to the rays is at least partially represented.

Simulations give the lens position, image positions and image amplifications corresponding to their energy fluxes. These parameters would also be observed in reality. The source position $\boldsymbol{\beta}_{\mathrm{S}}$ is unknown but it must be the same for all the images, and we use it as a model parameter. The source luminosity is also unknown, but the observed fluxes must be proportional to the lens amplifications in corresponding positions. Thus the model has to reproduce amplification ratios and not their absolute values. The intrinsic lens parameters (axis ratio $q$, characteristic deflection angle $\alpha_{0}$, the virial radius $r_{\mathrm{vir}}$, and the lens position angle in the sky $\phi$ ) are not so easy to measure and we treat them as unknown model parameters. Also the shear components $\gamma_{1}, \gamma_{2}$ belong to the class of intrinsic parameters. Below we use the value of shear defined as

$\gamma \equiv \sqrt{\gamma_{1}^{2}+\gamma_{2}^{2}}$

We treat the lens position, image positions and image fluxes ratios given by the simulations as observed quantities, each with its own uncertainty. We attempt to fit every simulated strong lensing case with our simplified model. We are going to reproduce the observed lens position, image positions and image flux ratios (or differences in image stellar magnitudes) looking for a minimum of $\chi^{2}$ taking all three constraints into account:

$\chi^{2}=\chi_{\mathrm{L}}^{2}+\chi_{\mathrm{I}}^{2}+\chi_{\mathrm{m}}^{2}$

The first term, controlling the lens position, used in the model has the obvious form:

$\chi_{\mathrm{L}}^{2}=\frac{\left(\boldsymbol{\beta}_{\mathrm{L}}-\boldsymbol{\beta}_{\mathrm{L}}^{0}\right)^{2}}{\sigma_{\mathrm{L}}^{2}}$

where the subscript L stands for "lens" and $\sigma_{\mathrm{L}}$ is the assumed accuracy of the lens position.

Using the simplified model we can calculate the source positions $\boldsymbol{\beta}_{\mathrm{S}}{ }_{\mathrm{S}}$ related to observed image positions $\boldsymbol{\beta}_{\mathrm{I}}{ }^{i 0}$. The deformation matrix $\mathrm{A}_{(i)}$ and magnification matrix $\mathrm{M}_{(i)}$ can be calculated at each image position:

$\boldsymbol{\beta}_{\mathrm{S}}^{i} \equiv \boldsymbol{\beta}_{\mathrm{S}}\left(\boldsymbol{\beta}_{\mathrm{I}}^{i 0}\right) \quad \mathrm{A}_{(i)} \equiv\left\|\frac{\partial \boldsymbol{\beta}_{\mathrm{S}}^{i}}{\partial \boldsymbol{\beta}_{\mathrm{I}}^{i 0}}\right\| \quad \mathrm{M}_{(i)} \equiv \mathrm{A}_{(i)}^{-1}$

The mismatch between $\boldsymbol{\beta}_{\mathrm{S}}^{i}$ and $\boldsymbol{\beta}_{\mathrm{S}}$ implies magnified mismatch between modeled and observed image positions, which gives for $\chi_{\mathrm{I}}^{2}$ (Kochanek 2006):

$\chi_{\mathrm{I}}^{2}=\sum_{i} \frac{\left|\mathrm{M}_{(i)} \cdot\left(\boldsymbol{\beta}_{\mathrm{S}}^{i}-\boldsymbol{\beta}_{\mathrm{S}}\right)\right|^{2}}{\sigma_{\mathrm{I}}^{2}}$

The fitting statistic for flux ratios is given as:

$\chi_{\mathrm{m}}^{2}=\sum_{i} \frac{\left(m^{i}-m^{0 i}-\left\langle m^{i}-m^{0 i}\right\rangle\right)^{2}}{\sigma_{\mathrm{m}}^{2}}$

In all cases the quantities with extra superscript " 0 " are taken from simulations and mimic the observed values while the quantities without this superscript are given by the model.

We use the following characteristic values for the accuracy parameters: $\sigma_{\mathrm{I}}=0.01 \operatorname{arcsec}, \sigma_{\mathrm{L}}=0.1 \operatorname{arcsec}$, and $\sigma_{\mathrm{m}}=0.1$ or 10 (in stellar magnitudes) This choice takes into account the fact that image positions are easier to measure than the lens position. With the small value of the flux measurement error $\left(\sigma_{\mathrm{m}}=0.1\right)$ we use the fluxes as important factor in modeling. The other value makes the fluxes unimportant in the model fitting. Using it one can check to which extent models well reproducing image positions are also good in reproducing ratios of their fluxes.

We start fitting process using a model with all parameter values, also the intrinsic lens parameters, taken from the simulation. This is not a general approach, but it is fast in finding solutions close to the starting point in parameter space (if they are present). 
Table 1. Acceptability of fits - dependence on the model

\begin{tabular}{ccccc}
\hline $\begin{array}{c}\text { Shear } \\
\sigma_{\mathrm{m}}\end{array}$ & no & no & yes & yes \\
10.1 & 10. & 0.1 & 10. \\
\hline Case 1 & $0.08 \pm 0.13$ & $0.11 \pm 0.13$ & $0.43 \pm 0.17$ & $0.68 \pm 0.22$ \\
Case 2 & $0.38 \pm 0.21$ & $0.41 \pm 0.18$ & $0.59 \pm 0.22$ & $0.78 \pm 0.19$ \\
Case 3 & $.9998 \pm .0002$ & $.9998 \pm .0001$ & $.9997 \pm .0002$ & $.9998 \pm .0001$ \\
\hline
\end{tabular}

Note: The table shows the dependence of the rate of acceptability of fits on the method of treating the light propagation (Cases 1-3, see the text for details), for models neglecting ("no") or taking into account ("yes") the external shear and reproducing $\left(\sigma_{\mathrm{m}}=0.1\right)$ or not $\left(\sigma_{\mathrm{m}}=10\right)$ the flux ratios. The assumed errors in fitting the positions of the lens center and of the images are kept constant $\left(\sigma_{\mathrm{L}}=0.1, \sigma_{\mathrm{I}}=0.01\right)$.

It is also our goal to obtain the "observable" parameters as close to simulated values as possible, but the intrinsic lens parameters are not constrained a'priori and have no preferred values.

Our approach to model fitting is not general, since we are probing only the small region of parameter space and there is a chance that we miss some acceptable models of the lens. On the other hand, if the influence of the environment were weak, the resulting lens model should be close to the original lens used in the simulations. Failure to find a model which is similar to the original can be interpreted as the result of the strong influence of the matter in the rays vicinity.

The number of the degrees of freedom (DOF) depends on the number of images $\left(N_{\mathrm{im}}\right)$ and on the number of intrinsic model parameters, which is $N_{\text {par }}=4$ for an elliptic lens $\left(q, \alpha_{0}, r_{\text {vir }}, \phi\right)$ and $N_{\text {par }}=6$ if the external shear $\left(\gamma_{1}, \gamma_{2}\right)$ is taken into account. The lens position is both observed and modeled, so it has no impact. The source position requires 2 parameters, and the source internal luminosity (which we do not use explicitly in modeling) - another one. When modeling the flux ratios with $\sigma_{\mathrm{m}}=0.1$ we effectively use the magnification information from the simulations, but for $\sigma_{\mathrm{m}}=10$ it is practically lost. In calculations we use: $D O F=3\left(N_{\mathrm{im}}-1\right)-N_{\mathrm{par}}$ (if $\left.\sigma_{\mathrm{m}}=0.1\right)$ or $D O F=2\left(N_{\mathrm{im}}-1\right)-N_{\mathrm{par}}$ (if $\left.\sigma_{\mathrm{m}}=10\right)$. (That implies that modeling of four-image configurations including shear and neglecting flux ratios is impossible, since $D O F=0$ in this case). Using tables of $\chi^{2}$ distribution we reject fits if they give

$\chi^{2}>\chi_{0.95}^{2}(D O F)$

where $\chi_{0.95}^{2}$ corresponds to $95 \%$ significance for a given $D O F$.

The influence of the matter distribution outside the lens on the lensing process can be characterized by the difficulty in obtaining an acceptable single lens model in such more complicated situations. To measure the rate of success in fitting procedure we assign the same weight to each investigated source position since they are distributed evenly in the source plane. Thus the more massive lenses have greater influence on the results as compared with less massive. This is the simplest consequence of the observational selection. More subtle effects, like magnification bias, influence of image configuration etc are too difficult to model within our approach.

Since we perform our simulations in eight different, randomly chosen regions of the synthetic Universe, we can estimate the role of the cosmic variance in our simulations. Calculating the rate of success for each of the regions separately and finding the dispersion of the results, we get an error estimate.

In Table. 1 we show the chances that a simulated strong lensing case has an acceptable simplified model. In Case 3 acceptable models are possible to obtain in almost $100 \%$ of simulated lens-
Table 2. Dependence of the acceptability of fits on required model accuracy

\begin{tabular}{ccccc}
\hline$\sigma_{\mathrm{I}}$ & 0.01 & 0.01 & 0.0025 & 0.0025 \\
$\sigma_{\mathrm{L}}$ & 0.1 & 0.025 & 0.1 & 0.025 \\
\hline Case 1 & $0.43 \pm 0.17$ & $0.39 \pm 0.18$ & $0.18 \pm 0.10$ & $0.16 \pm 0.10$ \\
Case 2 & $0.59 \pm 0.22$ & $0.47 \pm 0.18$ & $0.34 \pm 0.20$ & $0.28 \pm 0.17$ \\
Case 3 & $.9997 \pm .0002$ & $.9997 \pm .0002$ & $.9997 \pm .0001$ & $.998 \pm .001$ \\
\hline
\end{tabular}

Note: The table shows the dependence of the rate of acceptability of fits on the method of treating the light propagation (Cases 1-3, see the text for details) and on the assumed errors in measured positions of the center of the lens $\left(\sigma_{\mathrm{L}}\right)$ and of the images $\left(\sigma_{\mathrm{I}}\right)$. All models reproduce flux ratios $\left(\sigma_{\mathrm{m}}=0.1\right)$

image configurations, which shows that our fully numerical (used in obtaining lens - image configurations) and semi-analytical (used in fitting models) descriptions of the same physical approach are in complete agreement.

The results depend on the adopted values of position accuracy, (see below) but the difficulty in modeling realistic configurations using a single elliptical lens without external shear is apparent, even if the reproduction of correct flux ratios is not required. The inclusion of external shear substantially improves the chances of obtaining acceptable solutions with simplified lens models, but still there is no guarantee of success, especially if one requires the reproduction of the observed flux ratios.

The present day astrometry gives better accuracy of image positions than assumed in majority of our simulations. On the other hand the micro-lensing may change the measured fluxes of individual images in a random way, which diminishes their role as model constraints. In Table. 2 we show the results of our approach with increased required accuracy of positions of the lens, the images, or both, for models with shear, reproducing flux ratios.

In the following Fig. 3 we show the probability $P(>\gamma)$ that an acceptable fit uses external shear value greater than $\gamma$. The plots refer to fits taking into account image positions but not their flux ratios.

The influence of lens neighbours and matter in the lens foreground/background is also illustrated by the changes in the intrinsic lens parameters when comparing the fitted model with the original lens used in the simulation. In reality one has only one set of lens parameters obtained from modeling and nothing to compare with, nevertheless our plots show the likely systematic errors resulting from the model approximations.

In Fig. 4 we show (on a logarithmic graph) the integral distribution of the ratios of the fitted values of the deflection angle parameter to its original values used for the same lenses in simulations. Similarly Fig. 5 shows the distribution of the ratios between fitted and original virial masses.

\section{DISCUSSION AND CONCLUSIONS}

In simulations we replace each halo taken from the Millennium Simulation by a specific kind of matter distribution with the same mass and size, acting as non-singular isothermal ellipsoid with finite mass. We believe that this particular choice of lens model has little impact on our results, because our aim is to investigate the influence of the environment on the lens action, not the action itself. In particular a different choice of the relative size of the lens core (we always use $r_{1} / r_{2}=0.01$ ) may change the properties of 


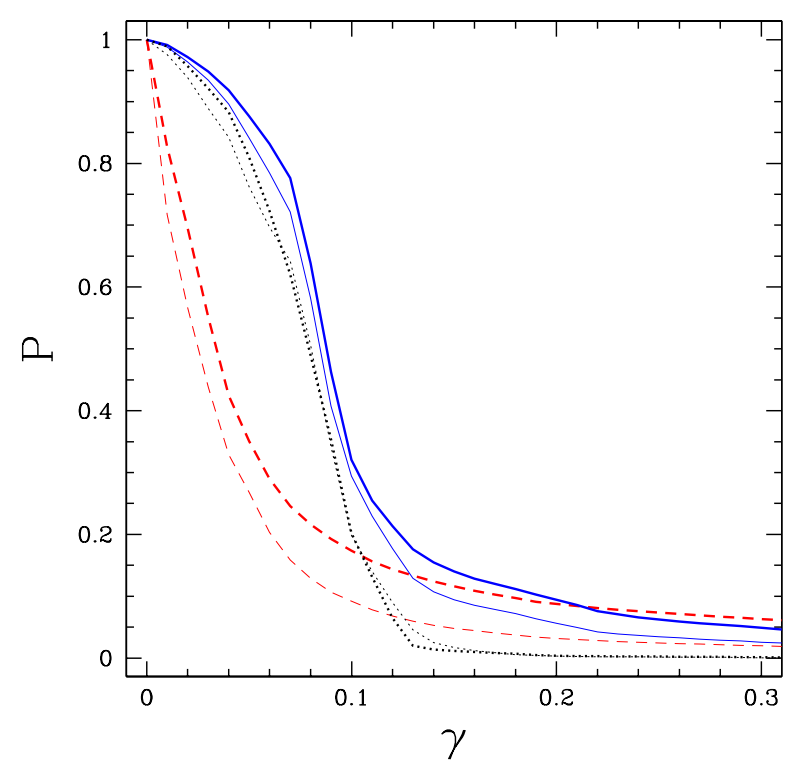

Figure 3. The integral probability distribution of the external shear value $\gamma$ for acceptable models. The results for models neglecting flux ratios $\left(\sigma_{\mathrm{m}}=\right.$ 10) in Case 1 are shown as solid lines (blue), and in Case 2 - as dashed lines (red). For comparison the results for models taking into account flux ratios $\left(\sigma_{\mathrm{m}}=0.1\right)$ in Case 1 are shown as dotted lines (black). The thick lines correspond to lower accuracy $\left(\sigma_{\mathrm{I}}=0.01, \sigma_{\mathrm{L}}=0.1\right)$ and the thin lines - to models of higher accuracy $\left(\sigma_{\mathrm{I}}=0.0025, \sigma_{\mathrm{L}}=0.025\right)$.

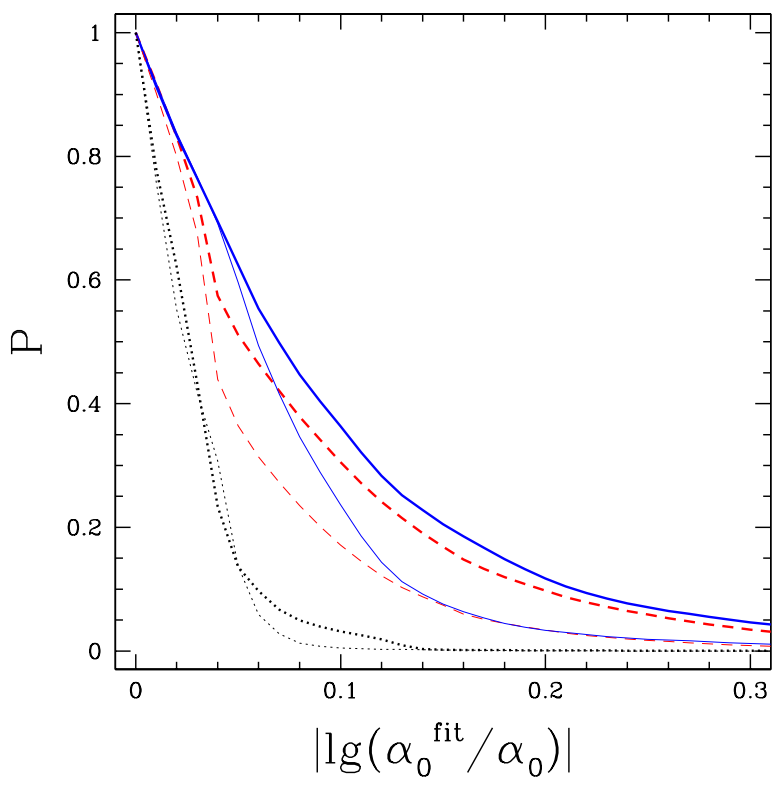

Figure 4. The integral probability distribution showing the errors in the fitted values of characteristics deflection angle $\alpha_{0}$. Conventions follow Fig. 3 $\alpha_{0}$ is the deflection angle of the lens used in simulations. $\alpha_{0}^{\text {fit }}$ is its fitted value.

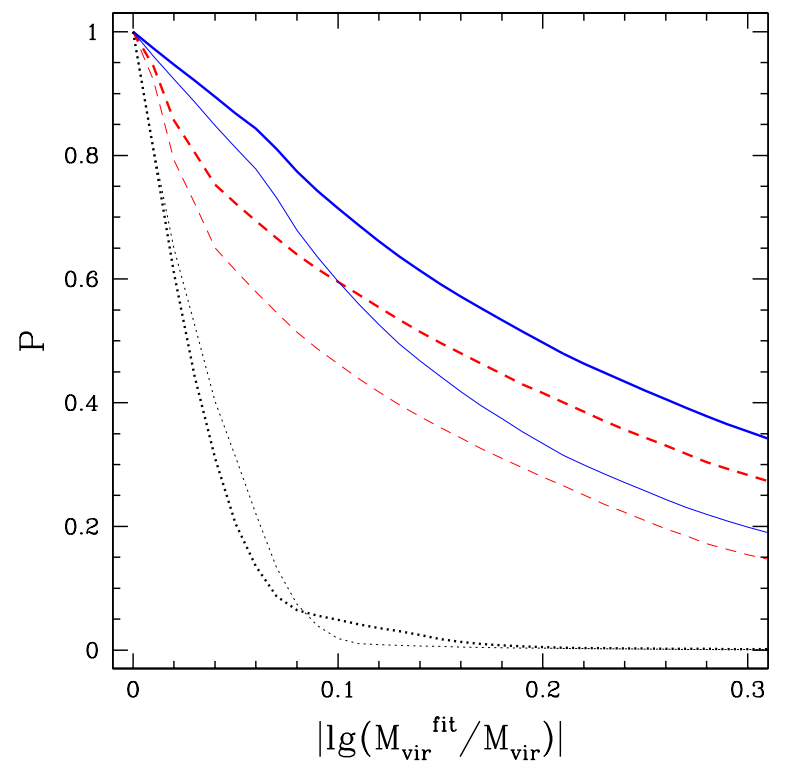

Figure 5. The integral probability distribution showing the errors in the fitted values of the lens virial mass. Line/colour conventions follow Fig. 3 $M_{\text {vir }}$ is the virial mass of the lens used in simulations. $M_{\text {vir }}^{\text {fit }}$ is its fitted value.

the radial caustic of each lens, but has no influence on the action of matter outside it.

We consider lenses with non-singular matter distribution so the number of images should be odd in all cases. In our calculations (which start from an approximate solution of lens equation) the solutions with four images are rare, 40 times less frequent than the five-image cases. The brightest images in our four-image configurations are on average brighter by $\approx 1.5^{\mathrm{mag}}$ as compared to the brightest images in five-image cases. Also the flux ratio of the brightest image to the next one is substantially higher (by $\approx 0.6^{\mathrm{mag}}$ ) in the four-image configurations. This suggests that our four-image solutions correspond to source positions close to the caustics, which produce close image pairs unresolvable by our approximate method of image finding. Since four-image configurations are rare, they have little impact on the statistics of the acceptability of fits.

The matter belonging to gravitationally bound haloes makes only a part of the whole matter distribution. The distribution of the unbound component we are using is known with limited resolution of $\approx 2 h^{-1} \mathrm{Mpc}$ in 3D. Since the influence of low density regions averages along the lines of sight on distances of several thousands megaparsecs, the averaging inside cells of few megaparsecs across probably has little meaning.

Our simulations cover eight small $\left(3^{\prime} \times 3^{\prime}\right)$, randomly chosen regions in the sky. In each of the regions we choose ten lenses, with largest Einstein ring for a source at $z \approx 2$ according to approximate, SIS lens formula. These are the strongest lenses in the region, giving the largest image separations and having the largest area closed by caustics in the source plane. That does not mean that every lensed source must be related to one of the lenses investigated, but for its random position in the region of interest this would happen in a vast majority of cases.

Our numerical experiment shows that matter close to the line of sight and matter in the close vicinity of the lens do have an important influence on its lensing properties. Our statement is of statistical nature: we have shown than in large part of image con- 
figurations obtained as a result of simulations taking into account the presence of matter concentrations near the lens it is impossible to obtain acceptable models neglecting their influence. The problem is more severe in case when simulations take into account not only lens close neighbours but also matter inhomogeneities along the beam. It shows that both close neighbours and matter along the line of sight are important. Our results are in general agreement with Wambsganss et al. 2004, 2005); D'Aloisio \& Natarajan (2011), but we address different aspect of the problem.

The quantitative characteristics of the effect depends on the required accuracy of the simplified modeling. It certainly depends on the source redshift as well, but we have not pursued this aspect of the problem, limiting ourselves to sources at $z_{\mathrm{s}} \approx 2$.

Some examples of the expected rate of success, when using simplified lens model to fit realistic image configurations, are shown in Tables. 1 and 2. We assume that every position of the source has the same probability. The selection of a sample of multiple image systems may also depend on the lens amplification, image configuration and other secondary issues, which are difficult to simulate and are neglected.

Our results in Tables. 1 and 2 take into account the fifth image, if present. In real cases the fifth images are not observed or very faint and difficult to use in modeling. We have performed some extra calculations neglecting the fifth images altogether. Of course it is always easier to model a less complicated configuration of images, so the rate of success is higher in this case. For instance the first column of Table. 1 would read: $0.16,0.48,0.9996$ (instead of $0.08,0.38,0.9998$ ) and the third column: $0.64,0.68,0.9995$ (instead of $0.43,0.59,0.9997)$. The changes are substantial, but do not qualitatively affect our conclusion.

Our calculations give typical values of the external shear, which is used by the acceptable simplified models. When modeling configurations resulting from the full approach (taking into account all matter inhomogeneities along the rays) we obtain median shear value $\gamma \approx 0.09$ (compare Fig. 3). For the single plane approach (which neglects lens foreground and background objects) we get $\gamma \approx 0.03$. Both numbers weakly depend on the required accuracy of modeling and do not change when the fifth image is neglected. Comparison of these two numbers and the inspection of Table. 1 suggest that shear produced by the matter along the beam and the matter in the lens vicinity cannot be neglected in majority of cases. It also shows that contribution to the shear by background and foreground objects is significant.

We have also compared the fitted values of lens parameters with the parameters of the lens used in the simulation. If the flux ratios are not modeled, the fitted parameters may substantially differ from their original values. For instance the characteristic bending angle of the lens $\alpha_{0}$ may differ by a factor greater than $\approx 1.2$ in $50 \%$ of cases (compare Fig. 4). This would imply similar errors in estimates of mass distribution in the central part of the lens. For the virial mass $\left(M_{\mathrm{vir}} \propto \alpha_{0} r_{\mathrm{vir}}\right)$ the discrepancies may exceed factor $\approx 1.5$ in $50 \%$ of cases (compare Fig. 5 ). The models reproducing also the flux ratios give much better accuracy of the intrinsic lens parameters fitting (dotted lines in Fig. 4, 5).

\section{ACKNOWLEDGMENTS}

We are grateful to the Anonymous Referee, whose critical remarks greatly improved the paper. The Millennium Simulation databases used in this paper and the web application providing on-line access to them were constructed as part of the activities of the German As- trophysical Virtual Observatory. We are grateful to Volker Springel for providing us with the smoothed Millennium density distribution in the early stage of this project. This work has been supported in part by the Polish National Science Centre grant N N203 581540.

\section{REFERENCES}

Bar-Kana R., 1996, ApJ, 468, 17

Bertone S., De Lucia G., Thomas P.A., 2007, MNRAS, 379, 1143

Carbone C., Matarrese S., 2008, MNRAS, 388, 1618

Chen J., Kravtsov A.V., Keeton C.R., 2003, ApJ, 592, 24

D'Aloisio A., Natarajan P., 2011, MNRAS, 411, 1628

De Lucia G., Blaizot J., 2007, MNRAS, 375, 2

Gunn J. E., Kristian J., Oke J. B., Westphal J. A. Young P. J., 1979, IAUC, 3431, 2

Hilbert S., Hartlap J., White S.D.M., Schneider P., 2009, A\&A, 499, 31

Jaroszynski M., Kostrzewa Z., 2010, AcA, 60, 41

Keeton C. R., Kochanek C. S., Seljak U., 1997, ApJ, 487, 42

Kochanek C.S., 2006, in: Gravitational Lensing: Strong, Weak and Micro, Saas-Fee Advanced Course 33, Springer, Berlin

Kochanek C.S., Apostolakis J., 1988, MNRAS, 235, 1073

Kormann R., Schneider P., Bartelmann M., 1994, A\&A, 284, 285

Lemson G. , Springel V., 2006, Astr.Soc.Pac.Conf., 351, 212

Puchwein E. , Hilbert S., 2009, MNRAS, 398, 1298

Schneider P., Ehlers J., Falco E.E., 1992, "Gravitational Lenses", Springer-Verlag Berlin Heidelberg New York

Schneider P., Weiss A., 1988, ApJ, 327, 526

Springel V., White S.D.M., Jenkins A., et al., 2005, Nature, 435, 629

Seitz S. Schneider P., 1992, A\&A, 265, 1

Walsh D., Carswell R.F., Weymann R.J., 1979, Nature, 279, 381

Wambsganss J., Bode P., Ostriker J.P., 2004, ApJ, 606, L93

Wambsganss J., Bode P., Ostriker J.P., 2005, ApJ, 635, L1

Young P., Gunn J. E., Oke J. B., Westphal J. A., Kristian J., 1981, ApJ, 244, 736 